\title{
Comportamiento al desgaste de fundiciones tipo "silal"
}

\author{
C. Camba*, Á. Varela*, V. Blázquez**, A. García*, J.L. Mier*y F. Barbadillo*
}

\begin{abstract}
Resumen Es este trabajo se han estudiado las propiedades tribológicas y mecánicas de cinco fundiciones con distintos contenidos de carbono y silicio. Cada fundición ha sido sometida a ensayos de dureza Brinell, y ensayos de resistencia al desgaste, según la norma ASTM G99, "Standard test method for wear testing with a pin-on-disk apparatus". Se han correlacionado los resultados obtenidos para estos ensayos con la microestructura, la composición y el proceso de colada de cada una de las fundiciones.
\end{abstract}

Palabras clave

Fundiciones grises de alta aleación; Silicio; Grafito esferoidal; Desgaste abrasivo; Pin on disk.

\section{Wear behaviour of "silal" cast irons}

\begin{abstract}
The tribological and mechanical properties of five cast-iron with different contents of carbon and silicon have been studied. Wear tests according ASTM G99 "Standard test method for wear testing with a pin-on-disk apparatus" and Brinell hardness measurements were carried out in every alloy. These results were related to microstructure, composition and casting processes of the cast irons.
\end{abstract}

Keywords

High-alloy graphitic irons; Silicon; Spheroidal graphite; Wear; Pin on disk.

\section{INTRODUCCIÓN}

Dentro del campo de las fundiciones aleadas existen las fundiciones de matriz ferrítica, de medio y alto contenido en silicio, conocidas con los nombres de "silal" y "durirón", respectivamente. En el caso del "silal" la aleación es resistente al calor y al hinchamiento, presenta una microestructura ferrítica con grafito interdendrítico, que es estable hasta la temperatura de transformación eutéctica y, debido al silicio disuelto en la ferrita, que la fragiliza, presenta baja tenacidad y resistencia mecánica. Asimismo, presenta poca resistencia al choque térmico. Tanto sus propiedades mecánicas como su resistencia a la oxidación se pueden mejorar, con la esferoidización del grafito, mediante adición de magnesio. La adición del molibdeno aumenta, a su vez, la resistencia a fluencia. Dichas fundiciones se suelen utilizar en parrillas de hornos, ceniceros y en componentes de turbinas de vapor para trabajos continuos o intermitentes, en rangos de temperaturas entre 650 y $825^{\circ} \mathrm{C}$. La sustitución parcial del silicio por el aluminio mejora la resistencia a la oxidación a alta temperatura y la resistencia mecánica ${ }^{[1-7]}$.

La fundición conocida con el nombre de "durirón" contiene más silicio pero menos carbono que el "silal", siendo su microestructura ferrítica, dado el carácter alfágeno del silicio. Presenta alta resistencia a la corrosión seca y a la corrosión por ácidos oxidantes, pero es mucho más frágil que el "silal", muy dura y difícilmente mecanizable, con resistencia a tracción y al choque inferior a la del "silal". La adición del molibdeno mejora la resistencia a la corrosión, pero no ocurre lo mismo con las propiedades mecánicas. Se utilizan para la fabricación de piezas que deban resistir la corrosión electroquímica.

\section{MÉTODO EXPERIMENTAL}

\subsection{Material ensayado}

Las fundiciones estudiadas en este trabajo han sido obtenidas en la planta piloto de fundición de la

\footnotetext{
* Escuela Politécnica Superior, Universidade da Coruña, C/ Mendizábal, s/n, 15403 Ferrol, A Coruña, España.

${ }^{* *}$ E.T.S.I.I. - U.P.M., C/ José Gutiérrez Abascal 2, 28006 Madrid, España.
} 
Escuela Técnica Superior de Ingenieros Industriales de la Universidad Politécnica de Madrid.

Se han obtenido cinco coladas diferentes, teniendo, cada una de ellas, un contenido en carbono y en silicio distinto. Cuatro de ellas se han fabricado adicionando $\mathrm{Ni}-\mathrm{Mg}$ y, una quinta, la "Fundición" 4, añadiendo cloruro de magnesio, en su lugar. Además, la "Fundición" 1 se coló con una cantidad de $\mathrm{Ni}-\mathrm{Mg}$ cinco veces inferior a la del resto de las muestras ${ }^{[8-10]}$.

\subsection{Procedimiento experimental}

Para determinar la composición química en carbono de las fundiciones se empleó un analizador de carbono LECO. El contenido en silicio se obtuvo siguiendo las pautas de la norma UNE 7-028-75, mediante el ataque de la muestra con diferentes ácidos para poder proceder a la separación, filtración y calcinación de la sílice. El contenido de silicio se determina por pérdida de peso en el proceso.

La dureza de las muestras se determinó mediante un durómetro Brinell, empleando como penetrador la bola de 2,5 $\mathrm{mm}$ de diámetro y aplicando una carga de $187,5 \mathrm{~kg}$.

Para medir la resistencia al desgaste de las muestras se siguieron las directrices de la norma ASTM G99, "Standard test method for wear testig with a pinon-disk apparatus" "11 y 12].

Se empleó, como pin abrasivo, una bola de carburo de wolframio con un diámetro de $4 \mathrm{~mm}$ y con una dureza de $75 \mathrm{HRC}$, que ejerció, sobre la superficie de la muestra a ensayar, una fuerza de $10 \mathrm{~N}$.

Las superficies ensayadas se prepararon, previamente a los ensayos de desgaste, con el fin de eliminar la influencia que sobre los resultados de los mismos tiene el acabado superficial, presentando, en todos los casos, una rugosidad media, $\mathrm{Ra}$, inferior a $1,5 \mu \mathrm{m}$.

Las muestras se sometieron a ciclos de desgaste, controlados a temperatura ambiente, que consistieron en un desgaste lineal total de $1.000 \mathrm{~m}$, a una velocidad de $0,25 \mathrm{~m} / \mathrm{s}$.

Durante el ensayo de desgaste se midió el coeficiente de fricción desarrollado entre la muestra y pin abrasivo, en tiempo real.

La resistencia al desgaste se calculó en función de la pérdida de masa experimentada por las diferentes muestras en cada ensayo.

Para completar el estudio, se llevó a cabo un análisis metalográfico de las cinco fundiciones, atacándolas con una mezcla del $20 \%$ de ácidd fluorhídrico, el $10 \%$ de ácido nítrico y el 70 \% de alcohol, después de la correspondiente preparación superficial.

\section{RESULTADOS Y DISCUSIÓN}

\subsection{Composición química de las fundiciones}

En la tabla I se muestran los resultados obtenidos para el contenido de carbono y de silicio de las distintas fundiciones.

Las tres primeras tienen contenidos en carbono muy similares, pero difieren en el contenido en silicio que varía casi en un $2 \%$, entre cada una de ellas.

La "Fundición" 4 es la de mayor contenido en carbono y el contenido en silicio que presenta tiene un valor intermedio con respecto a las anteriores, siendo la tercera más baja. Por último, la "Fundición" 5 tiene el contenido en carbono más bajo, pero es la de mayor contenido en silicio.

\subsection{Microestructura de las fundiciones}

En la figura 1 se puede observar la microestructura de la "Fundición" 1, donde se puede apreciar grafito esferoidal sobre matriz ferrítico-perlítica.

La figura 2 se corresponde con la microestructura de la "Fundición" 2 y, en ella, se puede apreciar, nuevamente, grafito, aunque en esta ocasión no forma esferas tan perfectas como en el caso anterior. La matriz sobre la que se encuentra el grafito, en este caso, también es de ferrita y perlita aunque la proporción de perlita que se observa es, considerablemente, menor.

En el caso de la "Fundición" 3, la microestructura se expone en la figura 3 y se puede observar una disposición muy similar a la de la figura 1 . Existe matriz, fundamentalmente, de perlita con contenidos menores de ferrita y, grafito esferoidal, en mayor proporción que en el caso de la "Fundición" 1.

Tabla I. Contenido en carbono y silicio de las muestras

Table I. Carbon and silicon content of the samples

\begin{tabular}{ccc}
\hline Muestra & C (\%) & Si (\%) \\
\hline "Fundición" 1 & 2,05 & 6,22 \\
"Fundición" 2 & 2,03 & 8,12 \\
"Fundición" 3 & 2,02 & 3,62 \\
"Fundición" 4 & 2,71 & 5,31 \\
"Fundición" 5 & 1,56 & 6,75 \\
\hline
\end{tabular}




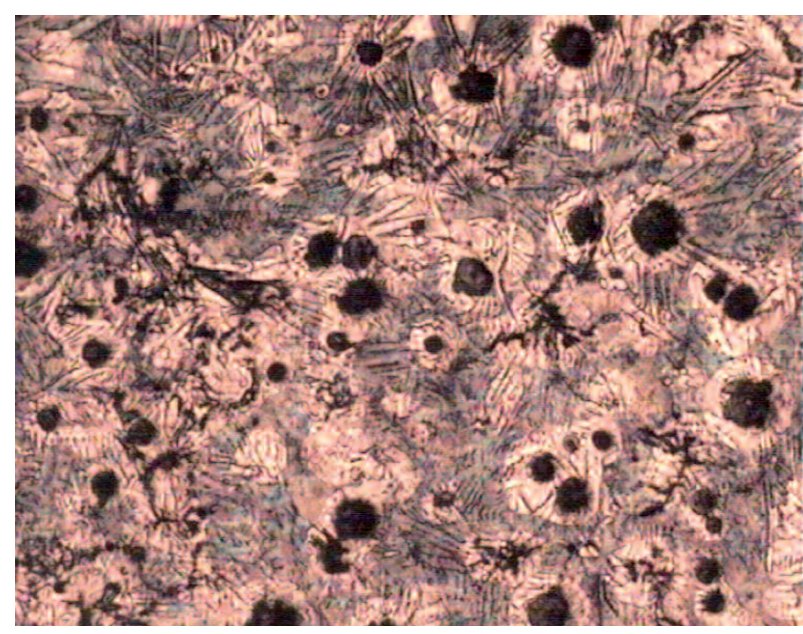

Figura 1. Microestructura de la "Fundición" 1 (X50).

Figure 1. Microstructure of the "Cast iron" 1 (X50).

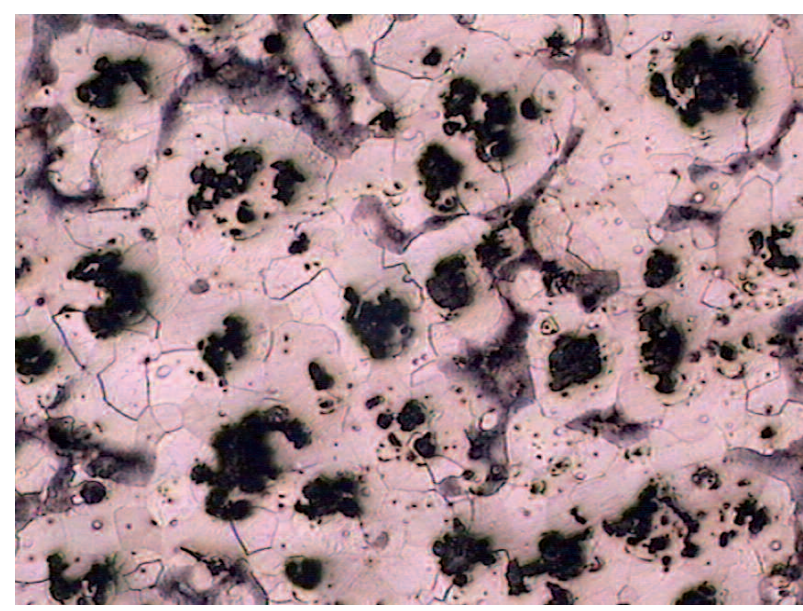

Figura 2. Microestructura de la "Fundición" 2 (X50).

Figure 2. Microstructure of the "Cast iron" 2 (X50).

Las "Fundiciones" 4 y 5 son las dos únicas muestras donde el grafito no está presente en forma esferoidal. La estructura, en forma de láminas de grafito, se ve perfectamente en la fotomicrografía correspondiente a la "Fundición" 4 (Fig. 4). En ambos casos, la matriz es, enteramente, ferrítica.

La principal diferencia entre la microestructura de ambas muestras es la disposición del grafito que, en el primer caso, aparece en forma perfectamente lam inar con distribución uniforme en toda la matriz, mientras que, en el segundo, el grafito es interdendrítico y su distribución no es tan uniforme, concentrándose, preferentemente, en el borde de grano (Fig. 5).

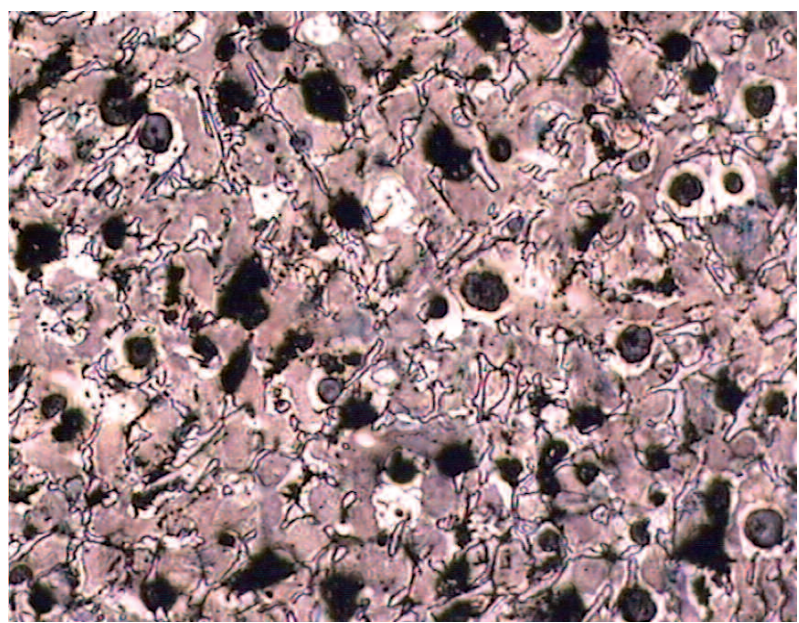

Figura 3. Microestructura de la "Fundición" 3 (X50).

Figure 3. Microstructure of the "Cast iron" 3 (X50).

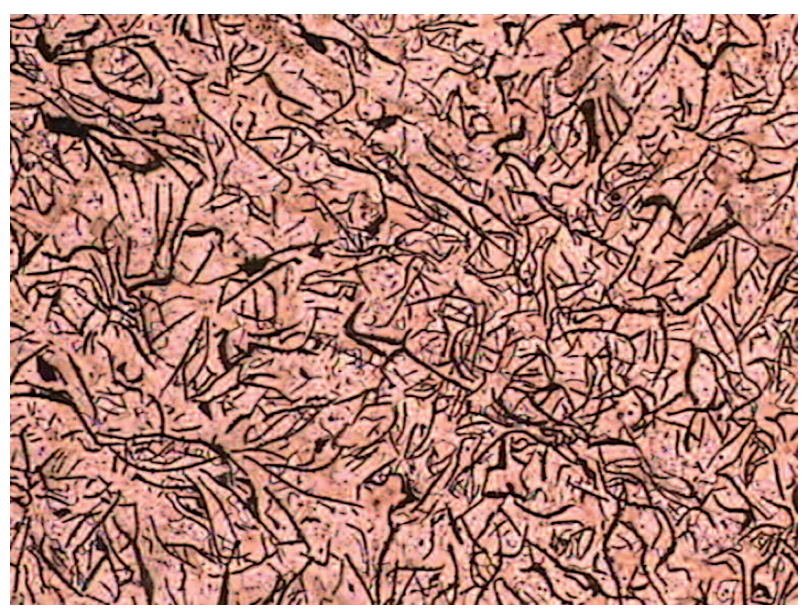

Figura 4. Microestructura de la "Fundición" 4 (X50).

Figure 4. Microestructure of the "Cast iron" 4 (X50).

\subsection{Dureza de las fundiciones}

En la tabla II se muestran los valores de dureza Brinell medidos para las cinco muestras estudiadas.

Según la tabla II, las "Fundiciones" 1 y 2 tienen un valor de dureza muy similar y algo inferior al que presenta la "Fundición" 3, que es la más dura de las cinco analizadas. El valor de dureza medido para la "Fundición" 5 es ligeramente inferior al de la 1 y la 2, mientras que la "Fundición" 4 es la que tiene el valor más bajo de todos. 


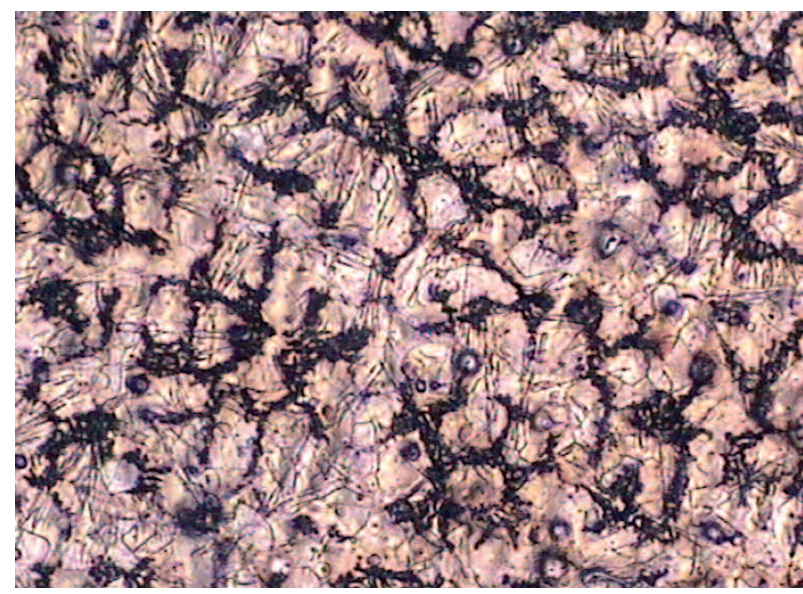

Figura 5. Microestructura de la "Fundición" 5 (X50).

Figure 5. Microstructure of the "Cast iron" 5 (X50).

Tabla II. Valores de dureza de las fundiciones

Table II. Hardness values of the cast irons

\begin{tabular}{cc}
\hline Muestra & HB \\
\hline "Fundición" 1 & 255 \\
"Fundición" 2 & 252 \\
"Fundición" 3 & 285 \\
"Fundición" 4 & 136 \\
"Fundición" 5 & 237 \\
\hline
\end{tabular}

\subsection{Resultados de desgaste}

En los ensayos de desgaste se ha determinado, por una parte, la resistencia al desgaste de las diferentes muestras en función de la pérdida de masa que experimentan durante el ensayo y, por otra, el coeficiente de fricción que se desarrolla entre la bola de carburo de wolframio que actúa como pin abrasivo y la muestra estudiada a lo largo de todo el ensayo de desgaste. En las figuras 6, 7 y 8 se muestran las gráficas del coeficiente de fricción obtenidas en tres ensayos, que sirven como ejemplo del patrón de comportamiento de las cinco fundiciones.

Como puede apreciarse en las figuras anteriores, existen tres patrones de comportamiento diferentes. En el primero (correspondiente a las "Fundiciones" 1 y 2), el coeficiente de fricción tarda muchos metros en alcanzar el valor definitivo y no se estabiliza hasta, casi, el final del ensayo, mientras que el patrón reflejado en la figura 7 (para las "Fundiciones" 3 y 5) muestra como el coeficiente de desgaste sube de manera

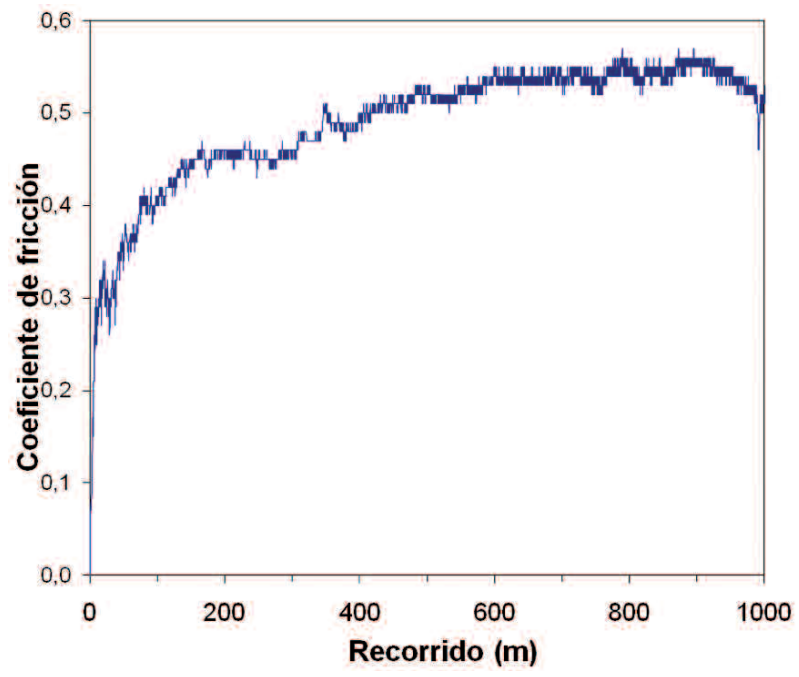

Figura 6. Patrón de comportamiento del coeficiente de fricción durante el ensayo de desgaste para las "Fundiciones" 1 y 2.

Figure 6. Friction coefficient evolution for wear tests of the "Cast irons" 1 and 2.

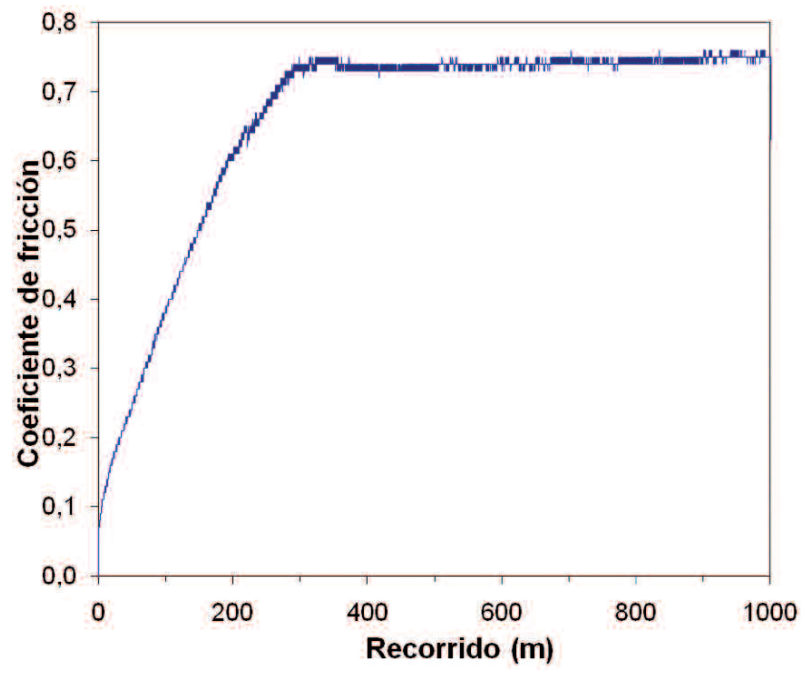

Figura 7. Patrón de comportamiento del coeficiente de fricción durante el ensayo de desgaste para las "Fundiciones" 3 y 5.

Figure 7 Friction coefficient evolution for wear tests of the "Cast irons" 3 and 5.

progresiva en el primer tercio del ensayo y, después, permanece, prácticamente, constante. Por último, el patrón que presenta la "Fundición" 4 implica una subida brusca del valor del coeficiente de fricción, desde el inicio del ensayo, alcanzando un valor estable, prácticamente, desde el principio del mismo.

El valor de coeficiente de fricción que se alcanza finalmente es más elevado para las muestras que 


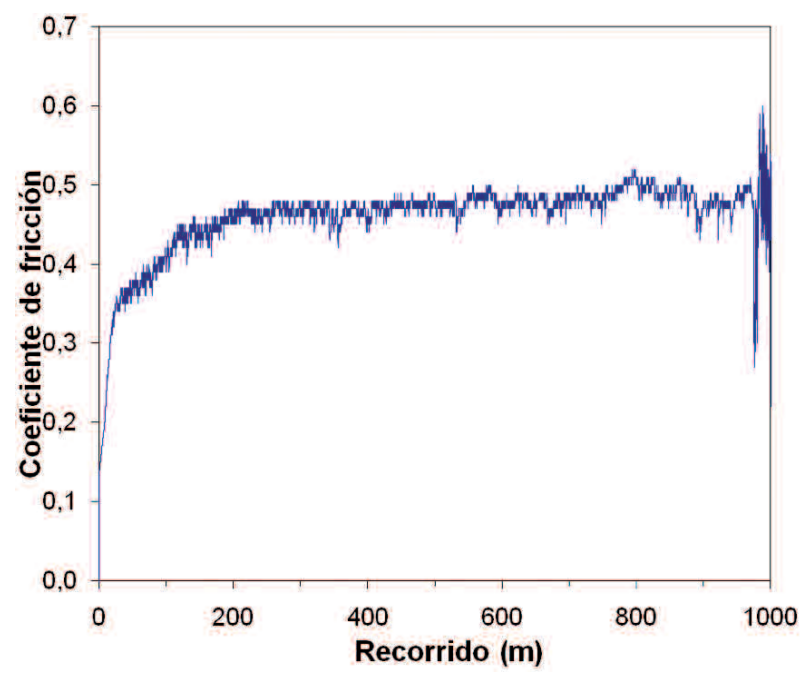

Figura 8. Patrón de comportamiento del coeficiente de fricción durante el ensayo de desgaste para la "Fundición" 4.

\section{Figure 8 Friction coefficient evolution for wear} test of the "Cast iron" 4.

siguen el patrón de comportamiento de la figura 7 ("Fundiciones" 3 y 5), mientras que las "Fundiciones" 1 y 2 (patrón de la figura 6) presentan valores intermedios y la "Fundición" 4 (Fig. 8) es la que se estabiliza en un valor inferior.

En la tabla III se muestran las pérdidas medias de masa obtenidas para cada una de las fundiciones ensayadas, junto con el coeficiente de variación obtenido para cada una de las muestras, en los distintos ensayos llevados a cabo. También, se incluye en la tabla III el coeficiente de fricción medio para cada una de las muestras.

Si se observan los resultados de pérdida de masa de la tabla III para las diferentes fundiciones, se aprecia cómo la "Fundición" 4 presenta un comportamiento marcadamente diferente al del resto de las

Tabla III. Resultados de desgaste

Table III. Wear results

\begin{tabular}{cccc}
\hline Muestra & $\begin{array}{c}\text { Pérdida de } \\
\text { masa (mg) }\end{array}$ & $\begin{array}{c}\text { Coeficiente } \\
\text { variación }\end{array}$ & $\begin{array}{c}\text { Coeficiente } \\
\text { fricción }\end{array}$ \\
\hline "Fundición" 1 & 5,39 & 0,06 & 0,49 \\
"Fundición" 2 & 3,00 & 0,07 & 0,58 \\
"Fundición" 3 & 1,89 & 0,08 & 0,62 \\
"Fundición" 4 & 36,13 & 0,12 & 0,46 \\
"Fundición" 5 & 1,74 & 0,14 & 0,66 \\
\hline
\end{tabular}

muestras analizadas, con una pérdida de masa un orden de magnitud superior. También, es la que presenta un coeficiente de fricción más bajo.

Las muestras que presentan un mejor comportamiento al desgaste son las "Fundiciones" 3 y 5, con una pérdida de masa muy semejante. Si se observa su coeficiente de fricción se puede ver cómo estas dos muestras son las que tienen un valor mayor.

Las "Fundiciones" 1 y 2 tienen un comportamiento algo peor que las dos anteriores, siendo algo mejor el de la "Fundición" 2. En cuanto a su coeficiente de fricción hay que decir que, de nuevo, la que más se desgasta, la "Fundición" 1, es la que tiene un coeficiente algo menor, aunque ambos valores también son intermedios entre los obtenidos para las fundiciones que mejor se comportan, la 3 y la 5 , y la que peor lo hace, la "Fundición" 4.

\subsection{Discusión}

En la figura 9 se presentan los resultados de dureza, resistencia al desgaste y coeficiente de fricción obtenidos para las cinco fundiciones.

Para poder representar el coeficiente de fricción con el resto de los parámetros se ha multiplicado por 50.

En esta figura, se puede observar cómo la resistencia al desgaste sigue un patrón semejante al de la dureza, de manera que, cuando ésta crece, la pérdida de masa que experimenta la muestra durante el ensayo de desgaste disminuye.

El mayor valor de la dureza se observa en la "Fundición" 3 que, como se vio en la figura 3, presenta una microestructura con matriz, fundamentalmente, perlítica y grafito esferoidal. Su microestructura es muy semejante a la que se observa en la figura 1, para la "Fundición" 1 que, sin embargo, pese a contener mayor proporción de silicio y, por tanto, ferrita más dura, tiene una dureza algo inferior a la de la "Fundición" 3. Esta disparidad entre el valor de dureza medido y el esperado, dada la microestructura, puede deberse a la diferencia en la cantidad de $\mathrm{Ni}-\mathrm{Mg}$ añadidos en la colada de las fundiciones, que es cinco veces superior, en el caso de la "Fundición" 3.

La "Fundición" 3 es la que presenta, junto con la 5, un comportamiento mejor frente al desgaste, a pesar de que, en ambos casos, el coeficiente de fricción medio es el más elevado de todos. Este comportamiento puede explicarse si se atiende a la figura 7, que representa el patrón de evolución seguido por el coeficiente de fricción durante los ensayos de desgaste, para estas muestras. Puede observarse que, aunque el valor del coeficiente que se alcanza al final del ensayo es mayor, durante el primer tercio del ensayo, este valor es mucho más bajo y va creciendo de 


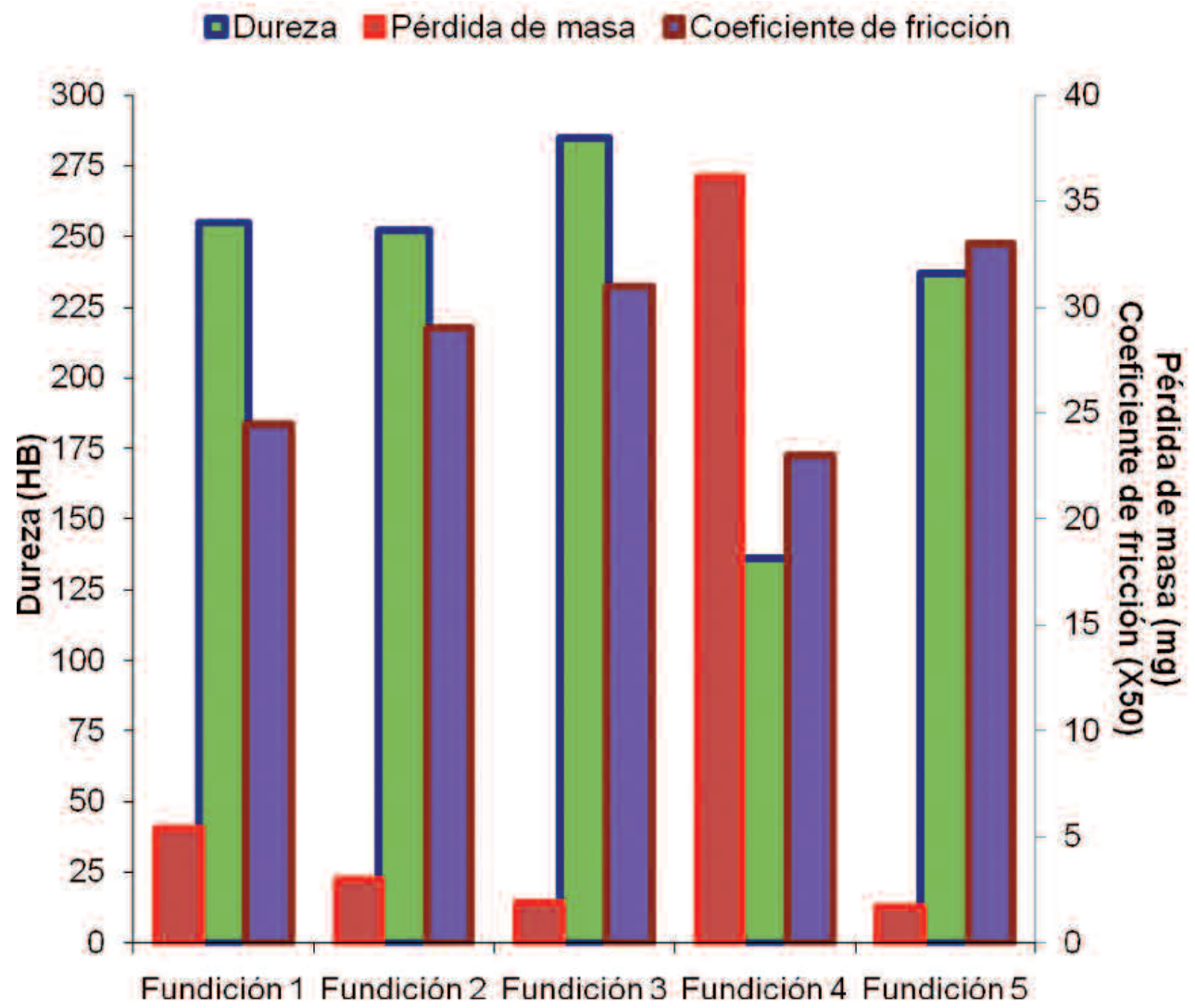

Figura 9. Resultados de desgaste y dureza obtenidos para las cinco fundiciones.

Figure 9. Wear and hardness results for the five cast irons.

manera progresiva. Puede concluirse que, durante esta etapa del ensayo y en ambos casos, el grafito presente está actuando como lubricante, lo que provoca esfuerzos de fricción mucho menores y pérdidas de masa, también, inferiores.

La muestra que presenta un comportamiento mucho peor al del resto es la "Fundición" 4, cuya pérdida de masa es, notablemente, mayor a la del resto de las muestras. En este caso, también, es la muestra con valor de dureza bastante inferior a las demás, lo que se justifica en virtud de la microestructura mostrada en la figura 4, donde se aprecia matriz enteramente ferrítica junto con grafito laminar. Esta disposición del grafito que, previsiblemente, se debe a la sustitución del $\mathrm{Ni}$-Mg por cloruro de magnesio en el proceso de colada, implica un mayor desprendimiento del mismo durante el ensayo de desgaste, lo que justifica una pérdida de masa superior a la del resto de las muestras. Además, en este caso, la pérdida del grafito ocurre desde el inicio del ensayo, por lo que el coeficiente de fricción que se obtiene es, prácticamente, constante a lo largo del mismo y no existe el efecto lubricación que aparecía para las "Fundiciones" 3 y 5 .
El comportamiento al desgaste de la "Fundición" 2 se justifica en función de su valor de dureza, inferior al de la "Fundición" 3. La pérdida de masa que experimenta durante el ensayo de desgaste es mayor que la de la "Fundición" 3, dado que la presencia de perlita en la matriz de la "Fundición" 2 es muy escasa. El valor de dureza de esta muestra es comparable al de la "Fundición" 1, lo que se puede justificar por su mayor contenido en silicio y, de nuevo, por la menor cantidad de $\mathrm{Ni}-\mathrm{Mg}$ añadido durante el proceso de colada, para la "Fundición" 1.

A pesar de presentar valores de dureza semejantes a los de la "Fundición" 1, su comportamiento frente al desgaste es peor, lo que se puede explicar por el mayor contenido de grafito que presenta en su microestructura y por la función lubricante del mismo, durante el ensayo. En este caso, la evolución del coeficiente de fricción, durante el proceso de desgaste, sigue el mismo patrón pero, si se analizan las curvas particulares de ambas muestras puede observarse cómo, en el caso de la "Fundición" 2, se tarda más en conseguir el valor definitivo del coeficiente, es decir, su tendencia es más próxima a la de las "Fundiciones" 3 y 5. 
El comportamiento más extraño lo presenta la "Fundición" 5. Tal y como se señaló, su comportamiento frente al desgaste es muy semejante al de la "Fundición" 3 y el valor de dureza que se ha medido es algo inferior a las medidas para las "Fundiciones" 1,2 y 3 . Sin embargo, la microestructura de esta fundición no hace predecir, a priori, este tipo de comportamiento. Esta fundición presenta matriz ferrítica y grafito interdendrítico, siendo la fundición de menor contenido en carbono y la única de las analizadas que presenta esta disposición del grafito.

El mayor valor de dureza de la "Fundición" 5 frente a la 4 se explica porque, a pesar de que, en ambos casos, la matriz presente es completamente ferrítica, en la "Fundición" 4 existe una mayor cantidad de grafito, por lo que el carbono en disolución es menor $y$, al ser el contenido en silicio muy semejante, la dureza de la "Fundición" 5 es superior.

\section{CONCLUSIONES}

Se ha estudiado el comportamiento mecánico, tribológico y la microestructura de cinco fundiciones con contenidos en carbono y silicio diferentes.

Cuatro de las fundiciones se han colado añadiendo $\mathrm{Ni}-\mathrm{Mg}$, mientras que, en la quinta, se ha añadido, en su lugar, cloruro de magnesio.

Se ha comprobado como el procedimiento de colada influye en las propiedades de la fundición, de forma que el peor comportamiento, tanto en lo que respecta a la dureza como en la resistencia al desgaste, se corresponde con la fundición colada con cloruro de magnesio que, además, es la única que presenta grafito en forma laminar. Además, a igualdad de microestructura, la fundición colada con menor proporción de $\mathrm{Ni}-\mathrm{Mg}$ obtiene peores resultados, en los ensayos realizados.
El mejor comportamiento frente al desgaste se corresponde con aquellas fundiciones en las que la cantidad o disposición del grafito favorece su papel como lubricante durante el ensayo.

\section{REFERENCIAS}

[1] V. Blázquez, Metalografía de las aleaciones férreas, Ed. Sección de Publicaciones E.T.S.I.I, Madrid, España, 1991, pp. 301-304.

[2] J.A. Pero-Sanz., Fundiciones férreas. Materiales para ingeniería, Ed. Dossat, Madrid, España, 1994, pp. 142-146.

[3] N.N. Aleksandrov y N.I. Klochnev, Production Technology and Properties of Heat-Resisting Cast Iron, Israel Program for Scientific Translations, Jerusalem, Israel, 1965, pp. 92-109.

[4] J.R. Davis, Cast Irons, Ed. ASM International Handbook Committee, Ohio, EE. UU., 1996, pp. 123-130.

[5] J. Apraiz, Fundiciones, Ed. Dossat, Madrid, España, 1986, pp. 143-146.

[6] W Fairhurt. y K.Roehrig, Rev. Foundry Trade 146 (1979) 657-681.

[7] K. Roehrig, Rev. Giesserei 61 (1974) 197-202.

[8] R.P. Walson, Trans. AFS, 85 (1977) 51.

[9] R. Gonzaga-Cinco y J. Fernández-Carrasquilla, Rev. Metal. Madrid 42 (2006) 91-102.

[10] A. Suárez-Sanabria y J. Fernández-Carrasquilla, Rev. Metal. Madrid 42 (2006) 18-31.

[11] ASTM Standard G99, Standard Test Method for Wear Testing with a Pin-on-Disk Apparatus, ASTM International, West Conshohocken, PA, California, EE. UU., 2008, pp. 408-412.

[12] J. A. Jiménez-Aparicio, A. López, O. Ruano y N. Candela, Rev. Metal. Madrid 41 (2005) 351 356. 